\title{
TREATMENT CHALLENGES IN ENTEROCOCCUS FAECIUM SEPSIS AND CLOSTRIDIUM DIFFICILE DIARRHEA IN A PATIENT WITH B-CELL NON-HODGKIN LYMPHOMA - CASE REPORT
}

\author{
Ramona Stefania Popescu ${ }^{1,2}$, Adina Ilie ${ }^{2}$, Rodica Bacruban ${ }^{2}$, \\ Catalin Apostolescu ${ }^{1,2}$, Adrian Streinu-Cercel ${ }^{1,2}$ \\ 1 "Carol Davila" University of Medicine and Pharmacy, Bucharest, Romania \\ 2 "Prof. Dr. Matei Bals" National Institute for Infectious Diseases, Bucharest, Romania
}

\begin{abstract}
The risk of developing secondary infections in immunocompromised patients due to hematological malignancies and the treatments for such conditions is very well known. Fever can be the only manifestation of a serious infection among this category of patients. The degree and duration of neutropenia is directly related to the risk of multiple infectious complications. When we are dealing with severely impaired host defenses virtually any microorganism can become invasive, bacteria (gram-positive pathogens, but gram-negative as well), being the greatest immediate threat.

Multiple pathogens isolated from an immunocompromised host can represent a major challenge for the clinician, especially when we have to face multidrug resistant (MDR) microorganisms, also called "superbugs". Besides, when we confront such a patient with many comorbidities and a high risk for MDR pathogens infection, we also confront with a lot of limitations in terms of treatment options.

We present a complicated case of glycopeptide-resistant Enterococcus faecium sepsis secondary to Clostridium difficile colitis in a 63 years old female patient with B-cell non-Hodgkin Lymphoma (NHL) during the R-CHOP therapy.
\end{abstract}

Keywords: sepsis, Enterococcus faecium, Clostridium difficile, fecal microbiota transplantation

\section{CASE REPORT}

A 63 years old female patient was admitted for watery diarrhea with mucus (more than 10 bowel movements a day), fever, nausea, diffuse abdominal pain, fatigue and anorexia, symptoms that had been present for more than one month. Her recent history revealed a B-cell non-Hodgkin lymphoma (NHL), diagnosed 6 months earlier for which she received five cycles of R-CHOP regimen (Rituximab, Cyclophosphamide, Doxorubicin, Vincristine, Prednisone). Also, during the past months the patient received several courses of antibiotics for nonspecific symptoms (Cefoperazonum + Sulbactamum, Penicillin and Gentamicin).

On examination, she was febrile, pale, hypotensive $(\mathrm{BP}=90 / 40 \mathrm{mmHg})$, tachycardic (pulse $=100$ bpm) with pain in her lower abdomen, peripheral edema and malaise. Laboratory tests showed mild leukocytosis $(\mathrm{WBC}=10.600$ per microL, 75\% neutrophyls), biological inflammatory syndrome, electrolyte imbalance, low albumin level. The stool studies for Salmonella, Sighella were negative, however polymerase chain reaction (PCR) for Clostridium difficile (CD) was positive for toxigenic 027 strain.

\section{Autor corespondent:}

Ramona Stefania Popescu, "Prof. Dr. Matei Bals" National Institute for Infectious Diseases, 1 Dr. Calistrat Grozovici Street, Bucharest

E-mail: ramona.stefania.popescu@gmail.com 
Based on these data we interpreted the case as C. difficile associated diarrhea (CDAD) induced by chemotherapy and we initiated treatment with orally Vancomycin $500 \mathrm{mg}$ q6hr. Under this therapy there was a slight clinical improvement, the patient became afebrile with a reduction in the number of stools. Unfortunately, after several days, still being under this treatment, the patient developed fever again with an increasing frequency of the stools. The blood culture obtained at this moment identified Enterococcus faecium - ampicillin, vancomycin, teicoplanin susceptible. Considering this, we ceased orally Vancomycin, started iv Teicoplanin $800 \mathrm{mg}$ loading dose then $400 \mathrm{mg}$ qd and for the resolution of CDAD we decided to perform fecal microbiota transplantation (FMT) from a healthy family member donor.

Despite antibiotic treatment according to antibiogram susceptibility and FMT for CDAD the patient failed to improve. She was still febrile having a great number of stools per day. At this point, we took into consideration the following possible causes of persistent fever and diarrhea: CD relapse, sepsis with different etiology or Enterococcus faecium vancomycin resistant sepsis with in vivo induced resistance after drug exposure. We ruled out $\mathrm{CD}$ relapse by testing the $\mathrm{CD} A / \mathrm{B}$ toxins witch came out negative. Subsequent blood cultures were also negative. We decided to switch teicoplanin to linezolid $600 \mathrm{mg} \mathrm{q12hr}$. After two days the patient became afebrile with progressive improvement in clinical status. She was discharged after ten days of iv linezolid, with normal stool consistency and overall very good condition.

Even if, we later failed to isolate an Enterococcus faecium strain vancomycin resistant, based on the rapid clinical improvement of the patient after switching teicoplanin to linezolid, we established that this was a case of Enterococcus faecium sepsis with in vivo induced resistance to vancomycin (after drug exposure) during the treatment for Clostridium difficile colitis.

\section{DISCUSSION}

Clostridium difficile is the most important cause of nosocomial infectious diarrhea in developed countries. $(1,2)$ Most CD strains with clinical relevance produce both toxin $A$ (enterotoxin) and toxin B (cytotoxin). Only toxin-producing strains are pathogenic. (3)

Due to increasing incidence and severity, CD infections (CDI) are becoming an important public health issue. The clinical picture ranges from as- ymptomatic carriage to self-limiting diarrhea or pseudomembranous colitis with toxic megacolon witch associates a very poor prognosis. Nowadays, CD represents an important cause of hospital-acquired infection, but as well as healthcare-associated disease (4); furthermore, community-associated disease are more frequently reported with an incidence that tends to reach levels equal to hospitalacquired disease. (5) The CDI mortality rate has been increasing over the last decade, due to the development of hypervirulent and antibiotic-resistant strains, such as ribotype 027. (6) In an European hospital-based survey of CDI, the prevalence of ribotype 027 was $5 \%$ (7), the most prevalent were ribotypes 014/020, 001, and 078. (7)

Frequent use of antibiotics has been shown to drastically reduce and alter the composition of preexisting host microbiome, these modifications in host microbiota is clearly illustrated in the setting of CDI. This allows the proliferation of the pathogen and the production of toxins that cause diarrhea and colonic disease. (8) Several studies have demonstrated less diversity in the colonic microbiota in CDI and recurrent CDI patients compared with those in healthy individuals. $(8,9)$

Previous use of antibiotics is the most important risk factor for CDI (10), particularly the use of clindamycin (11), cephalosporins (12) and flouroquinolones. (13) The risk is highest during or within the first month after antibiotic use. (14) Other established risk factors include comorbidity, recent hospitalization, advanced age ( $>65$ years), immunosuppression and proton pump inhibitor use. $(7,15)$ Those with serious CDI often have a high degree of comorbidity and long exposure to antibiotics. (16)

Currently, the treatment guidelines for mild to moderate $\mathrm{CD}$ infection include metronidazole or vancomycin. (10) However, the cure rates of severe CDI treated with metronidazole and vancomycin are only $76 \%$ and $97 \%$, respectively. Within 8 weeks the chance of recurrent CDAD is $10-20 \%$, but once a patient develops recurrence, the rate of further recurrences increases to $40-65 \%$. (17) Oral metronidazole is the normal first-line treatment, while vancomycin is used in more severe cases or with metronidazole failure. (15) Tapered or pulsedosage vancomycin can be used to reduce the risk of a subsequent recurrence.

Fidaxomicin is a recently introduced antibiotic with activity against $C$. difficile, recommended for the treatment of an initial episode of severe CDI as well as for recurrences (18) and shows equal efficacy compared to vancomycin. (19) 
All antibiotics currently in use for CDI have major limitations: high recurrence, risk of resistance development, and intestinal dysbiosis. Metronidazole has almost no effect in severe cases, with reduced susceptibility being observed in $C$. difficile ribotypes 001. (20) Vancomycin use is associated with increasing risk of induced vancomycin resistance among Enterococcus spp. (21) Fidaxomicin has limited therapeutic efficacy for the hypervirulent epidemic strains of ribotype 027. (22)

Cadazolid, a novel oxazolidinone antibiotic, currently in trials, has recently shown potent antimicrobial activity against $C$. difficile and has a lower propensity to induce resistance (18) or dysbacteriosis.

However, because recurrence rates are still relatively high, it is necessary to look into alternative therapies for CDI. (15) In this regard, considering the effective outcome, relative low cost, apparent safety and availability of fecal microbiota transplantation (FMT), it has become an increasingly accepted option for treatment of CDAD. (23) FMT is a procedure used to restore the intestinal microbiota of an individual using indigenous intestinal microorganisms from a healthy donor. The fecal filtrate can then be delivered by oral capsule, nasogastric tube, colonoscopy or rectal enema. FMT are commonly used to treat patients with CDI refractory to standard antibiotic therapy (24), with a success rate reported to be $>90 \%$. (24)

Human fecal microbiota is composed of four main groups of bacteria (phyla), namely Firmicutes, Bacteroidetes, Proteobacteria and Actinobacteria (25), of those the first two groups account for more than $80 \%$ of the microbiota. Firmicutes comprise mostly Gram-positive bacteria with a DNA that has a low $\mathrm{G}+\mathrm{C}$ content, but also include Gram-negative bacteria. Bacteroidetes include Gram-negative bacteria, with the main representative being Bacteroides genus in the human gut. Proteobacteria consist of Gram-negative bacteria, many of them are wellstudied pathogens. Actinobacteria are a group of Gram-positive bacteria with a DNA that has a high $\mathrm{G}+\mathrm{C}$ content. (25)

Vancomycin reduced fecal microbial diversity with a decrease of gram-positive bacteria (mainly Firmicutes) and a compensatory increase in gramnegative bacteria (mainly Proteobacteria) (26), providing a selective advantage for vancomycin resistant enterococci (VRE) that may be present in small numbers in the individual's bowel. (27) Fidaxomicin has reduced effects on the commensal gut microbiota compared to vancomycin. Importantly, there was also decreased CDI recurrence rates in patients taking fidaxomicin compared to vancomycin, highlighting its role as a superior alternative antibiotic. $(19,28)$

Unlike antibiotic treatment for CDI, which maintain an abnormal colon microbiota, setting up a cycle of repeating infections, called recurrent CDI, FMT has the main goal to reestablish a diverse, "normal" microbiome within the colon. (29)

Enterococci have evolved over the past few decades from being harmless intestinal commensals of little clinical significance to becoming the second or third most common pathogens associated with nosocomial infection. (30) The most common human pathogens among various species of Enterococcus are E. feacalis and E. faecium. The latter as compared to E. faecalis display a higher degree of drug resistance to multiple other antibiotics, including ampicillin, gentamicin, ciprofloxacin, vancomycin and teicoplanin. $(31,32)$ Failure to recognize the resistant strains may result in inadequate antibiotic therapy with its associated morbidity and mortality.

Even if, earlier reports (33) showed failure to develop vancomycin-resistant Enterococcus with oral vancomycin treatment of CDI, Cobrun et al reported (34) the emergence of vancomycin resistance following exposure to vancomycin in a patient colonized with a isolate of E. faecium which was initially vancomycin susceptible.

On this basis, we cautiously conclude that in the setting of Enterococcus faecium sepsis and CDAD the FMT was the best choice of treatment for our patient.

\section{CONCLUSION}

The case brings again to attention the risk of developing vancomycin-resistant Enterococcus after oral therapy for CDI. Also, it points out that in difficult to treat CDAD, in our case due to secondary sepsis with E. faecium, FMT represents a reliable option easy to access and perform, with almost immediate results and very low risk of recurrences.

\section{Acknowledgement}

This paper is partially supported by the Sectoral Operational Programme Human Resources Development (SOP HRD), financed from the European Social Fund and by the Romanian Government under the contract number POSDRU/159/1.5/S/ 137390 . 


\section{REFERENCES}

1. Barbut F., Corthier G., Charpak Y., Cerf M., Monteil H., Fosse T., et al. Prevalence and pathogenicity of Clostridium difficile in hospitalized patients. A French multicenter study. Archives of internal medicine. 1996; 156(13):1449-54.

2. Johnson S., Gerding D.N. Clostridium difficile-associated diarrhea. Clinical infectious diseases: an official publication of the Infectious Diseases Society of America. 1998; 26(5):1027-34; quiz 35-6.

3. Kelly C.P., Pothoulakis C., LaMont J.T. Clostridium difficile colitis. The New England journal of medicine. 1994; 330(4):257-62.

4. Price M.F., Dao-Tran T., Garey K.W., Graham G., Gentry L.O., Dhungana L., et al. Epidemiology and incidence of Clostridium difficile-associated diarrhoea diagnosed upon admission to a university hospital. The Journal of hospital infection. 2007; 65(1):42-6.

5. Kuntz J.L., Chrischilles E.A., Pendergast J.F., Herwaldt L.A., Polgreen P.M. Incidence of and risk factors for communityassociated Clostridium difficile infection: a nested case-control study. BMC infectious diseases. 2011; 11:194.

6. He M., Miyajima F., Roberts P., Ellison L., Pickard D.J., Martin M.J., et al. Emergence and global spread of epidemic healthcareassociated Clostridium difficile. Nature genetics. 2013; 45(1):109-13.

7. Bauer M.P., Notermans D.W., van Benthem B.H., Brazier J.S., Wilcox M.H., Rupnik M., et al. Clostridium difficile infection in Europe: a hospital-based survey. Lancet. 2011; 377(9759):63-73.

8. Khoruts A., Dicksved J., Jansson J.K., Sadowsky M.J. Changes in the composition of the human fecal microbiome after bacteriotherapy for recurrent Clostridium difficile-associated diarrhea. Journal of clinical gastroenterology. 2010; 44(5):354-60.

9. Chang J.Y., Antonopoulos D.A., Kalra A., Tonelli A., Khalife W.T., Schmidt T.M., et al. Decreased diversity of the fecal Microbiome in recurrent Clostridium difficile-associated diarrhea. The Journal of infectious diseases. 2008; 197(3):435-8.

10. Cohen S.H., Gerding D.N., Johnson S., Kelly C.P., Loo V.G., McDonald L.C., et al. Clinical practice guidelines for Clostridium difficile infection in adults: 2010 update by the society for healthcare epidemiology of America (SHEA) and the infectious diseases society of America (IDSA). Infection control and hospital epidemiology. 2010; 31(5):431-55.

11. Slimings C., Riley T.V. Antibiotics and hospital-acquired Clostridium difficile infection: update of systematic review and meta-analysis. The Journal of antimicrobial chemotherapy. 2014; 69(4):881-91.

12. Nelson D.E., Auerbach S.B., Baltch A.L., Desjardin E., BeckSague C., Rheal C., et al. Epidemic Clostridium difficile-associated diarrhea: role of second- and third-generation cephalosporins. Infection control and hospital epidemiology. 1994; 15(2):88-94.

13. Pepin J., Saheb N., Coulombe M.A., Alary M.E., Corriveau M.P., Authier S., et al. Emergence of fluoroquinolones as the predominant risk factor for Clostridium difficile-associated diarrhea: a cohort study during an epidemic in Quebec. Clinical infectious diseases: an official publication of the Infectious Diseases Society of America. 2005; 41(9):1254-60.

14. Hensgens M.P., Goorhuis A., Dekkers O.M., Kuijper E.J. Time interval of increased risk for Clostridium difficile infection after exposure to antibiotics. The Journal of antimicrobial chemotherapy. 2012; 67(3):742-8.

15. Schenck L.P., Beck P.L., MacDonald J.A. Gastrointestinal dysbiosis and the use of fecal microbial transplantation in infection. World journal of gastrointestinal pathophysiology. 2015; 6(4):169-80.

16. Byrn J.C., Maun D.C., Gingold D.S., Baril D.T., Ozao J.J., Divino C.M. Predictors of mortality after colectomy for fulminant Clostridium difficile colitis. Archives of surgery. 2008; 143(2):150-4; discussion 5.

17. Surawicz C.M., Brandt L.J., Binion D.G., Ananthakrishnan A.N., Curry S.R., Gilligan P.H., et al. Guidelines for diagnosis, treatment, and prevention of Clostridium difficile infections. The American journal of gastroenterology. 2013; 108(4):478-98; quiz 99.
18. Kali A., Charles M.V., Srirangaraj S. Cadazolid: A new hope in the treatment of Clostridium difficile infection. The Australasian medical journal. 2015; 8(8):253-62.

19. Mullane K.M., Miller M.A., Weiss K., Lentnek A., Golan Y., Sears P.S., et al. Efficacy of fidaxomicin versus vancomycin as therapy for Clostridium difficile infection in individuals taking concomitant antibiotics for other concurrent infections. Clinical infectious diseases: an official publication of the Infectious Diseases Society of America. 2011; 53(5):440-7.

20. Baines S.D., O'Connor R., Freeman J., Fawley W.N., Harmanus C., Mastrantonio P., et al. Emergence of reduced susceptibility to metronidazole in Clostridium difficile. The Journal of antimicrobial chemotherapy. 2008; 62(5):1046-52.

21. Goudarzi M., Seyedjavadi S.S., Goudarzi H., Mehdizadeh Aghdam E., Nazeri S. Clostridium difficile Infection: Epidemiology, Pathogenesis, Risk Factors, and Therapeutic Options. Scientifica. 2014; 2014:916826.

22. Lubbert C., John E., von Muller L. Clostridium difficile infection: guideline-based diagnosis and treatment. Deutsches Arzteblatt international. 2014; 111(43):723-31.

23. Brandt L.J., Aroniadis O.C., Mellow M., Kanatzar A., Kelly C., Park T., et al. Long-term follow-up of colonoscopic fecal microbiota transplant for recurrent Clostridium difficile infection. The American journal of gastroenterology. 2012; 107(7):1079-87.

24. Mittal C., Miller N., Meighani A., Hart B.R., John A., Ramesh M. Fecal microbiota transplant for recurrent Clostridium difficile infection after peripheral autologous stem cell transplant for diffuse large B-cell lymphoma. Bone marrow transplantation. 2015; 50(7):1010.

25. Panda S., El khader I., Casellas F., Lopez Vivancos J., Garcia Cors M., Santiago A., et al. Short-term effect of antibiotics on human gut microbiota. PloS one. 2014; 9(4):e95476.

26. Vrieze A., Out C., Fuentes S., Jonker L., Reuling I., Kootte R.S., et al. Impact of oral vancomycin on gut microbiota, bile acid metabolism, and insulin sensitivity. Journal of hepatology. 2014; 60(4):824-31.

27. Cetinkaya Y., Falk P., Mayhall C.G. Vancomycin-resistant enterococci. Clinical microbiology reviews. 2000; 13(4):686-707

28. Louie T.J., Miller M.A., Mullane K.M., Weiss K., Lentnek A., Golan Y., et al. Fidaxomicin versus vancomycin for Clostridium difficile infection. The New England journal of medicine. 2011; 364(5):422-31.

29. Bowman K.A., Broussard E.K., Surawicz C.M. Fecal microbiota transplantation: current clinical efficacy and future prospects. Clinical and experimental gastroenterology. 2015; 8:285-91.

30. Gb S., et al. Neonatal septicaemia caused by vancomycin resistant enterococcus faecium-a case report. Journal of clinical and diagnostic research: JCDR. 2014; 8(11):DD03-4.

31. Murray B.E. Vancomycin-resistant enterococci. The American journal of medicine. 1997; 102(3):284-93.

32. Udo E.E., Al-Sweih N., Phillips O.A., Chugh T.D. Species prevalence and antibacterial resistance of enterococci isolated in Kuwait hospitals. Journal of medical microbiology. 2003; 52(Pt 2):163-8.

33. Salgado C.D., Giannetta E.T., Farr B.M. Failure to develop vancomycin-resistant Enterococcus with oral vancomycin treatment of Clostridium difficile. Infection control and hospital epidemiology. 2004; 25(5):413-7.

34. Coburn B., Low D.E., Patel S.N., Poutanen S.M., Shahinas D., Eshaghi A., et al. Vancomycin-variable Enterococcus faecium: in vivo emergence of vancomycin resistance in a vancomycinsusceptible isolate. Journal of clinical microbiology. 2014; 52(5):1766-7. 